\title{
Boulonnage à ancrage réparti : étude de l'interface barre-scellement sous sollicitations axiales
}

\author{
Laura Blanco-Martín ${ }^{1, \star}$, Michel Tijani ${ }^{1}$, Faouzi Hadj-Hassen ${ }^{1}$ et Aurélien Noiret ${ }^{2}$ \\ ${ }^{1}$ Centre de Géosciences MINES-ParisTech, 35 rue Saint-Honoré, 77300 Fontainebleau cedex, France \\ 2 Andra, Laboratoire de recherche souterrain de Meuse/Haute-Marne, RD 960, BP 9, 55290 Bure, France
}

\begin{abstract}
Résumé - Dans cet article, nous étudions l'interface barre-scellement du boulonnage/câblage à ancrage réparti. L'application d'un effort de traction sur la barre permet d'examiner le phénomène de décohésion de l'interface. Des études expérimentales et théoriques ont été menées, en conditions statiques. Les études théoriques sont utiles pour convertir les variables observables dans les matériaux environnants (barre, scellement, terrain) en variables d'interface, non accessibles directement. Les études expérimentales, effectuées en laboratoire et in situ, permettent de déterminer les facteurs les plus influents sur la réponse de l'interface. La combinaison de ces deux études constitue une méthode d'accès à la réponse de l'interface barre-scellement. Un exemple d'application de la nouvelle méthode est détaillé pour une configuration en laboratoire. L'analyse de plusieurs interfaces suggère que la réponse tangentielle n'est pas seulement due au frottement et que la réponse radiale dépend fortement du profil de la barre.
\end{abstract}

Mots clés : Boulonnage à ancrage réparti / interface barre-scellement / approche analytique / essai d'arrachement / formulation semi-empirique / modèle d'interface

\begin{abstract}
Fully grouted bolts: assessment of the bolt-grout interface response under axial loads. In this paper, we investigate the bolt-grout interface of fully grouted bolts. To study the interface debonding process, an axial tensile load is applied on the bar. Experimental and theoretical investigations have been conducted, in static conditions. The theoretical studies allow converting the variables measured in the surrounding materials (bolt, grout, ground) in interface variables, which cannot be measured directly. The experimental studies have been carried out at laboratory and field scales. They are useful to determine the most influencing parameters on the interface response. The combination of the theoretical developments with the experimental results leads to a method to access the bolt-grout interface response. An application example of the new method is detailed for a laboratory set-up. The analysis of several interfaces suggests that the tangential response is not only due to friction and that the normal response is highly dependent on the bolt profile.
\end{abstract}

Keywords: Fully grouted bolts / bolt-grout interface / analytical approach / pull-out test / semi-empirical formulation / interface model

\section{Introduction}

Le boulonnage et le câblage à ancrage réparti sont deux techniques de soutènement couramment utilisées dans l'industrie minière et dans le génie civil. D'après Stillborg (1986), le boulonnage et le câblage sont les techniques de renforcement du terrain les plus efficaces et économiques. Les principaux avantages du boulonnage-câblage sont : une installation facile et mécanisable, un encombrement très réduit dans la section droite de l'ouvrage, un coût faible par rapport à d'autres techniques de soutènement, une souplesse pour être combiné à d'autres techniques et une capacité pour soutenir de grands espaces souterrains (Fine, 1998 ; Stillborg, 1986 ; Tincelin et Fine, 1991). Pour qu'un système de renforcement par boulonnage-câblage soit efficace, le temps de pose vis-à-vis

\footnotetext{
* Auteur correspondant :

laura.blanco_martin@mines-paristech.fr
}

de la déformation du terrain est très important. Ce type de renforcement peut être utilisé pour des applications aussi bien temporaires que permanentes.

Le principe du boulonnage et du câblage est le même (de fait, on tend à utiliser le terme " boulonnage » indistinctement) : on fore un trou dans le terrain, on y insère une tige et on l'ancre de façon à ce qu'elle soit solidaire du terrain environnant. La principale différence entre le boulonnage et le câblage est la nature de la barre employée : les boulons sont des tiges (lisses ou crénelées, en acier ou en fibres de verre), tandis que les câbles sont composés d'un nombre de torons en acier plus ou moins torsadés autour d'un fil central. Comme les boulons, les torons peuvent être lisses ou crénelés (pour améliorer la qualité de l'ancrage). D'après Windsor et Thompson (1993), le boulonnage à ancrage réparti est composé de quatre éléments principaux : la barre (boulon ou câble), le terrain environnant (roche ou sol), la fixation externe à la surface de l'excavation (plaque fixée par un écrou généralement) et la fixation interne à la paroi du trou borgne (matériau 
de scellement). L'ancrage entre la barre et le terrain environnant est effectué sur toute la longueur de la barre et dans la plupart des cas, le matériau de scellement employé est préparé à base de résine ou de ciment. Le boulonnage à ancrage réparti est un type de soutènement passif, car le boulon n'est activé que si le terrain environnant vient à se déformer. Par ailleurs, étant donné que toute la longueur de la barre est ancrée dans le matériau de scellement, le risque de corrosion des boulons à ancrage réparti est bien inférieur à celui des boulons à ancrage ponctuel ou à friction.

Les boulons à ancrage réparti peuvent supporter des efforts axiaux (traction et compression), des efforts de cisaillement et des moments de flexion. En conséquence, ce type de boulon est souvent soumis à des chargements complexes avant la rupture. Dans le but de mieux maîtriser le processus de transfert de charge entre le soutènement et le terrain, le comportement des interfaces barre-scellement et scellement-terrain doit être bien compris. Dans cette recherche, on s'intéresse à l'interface barre-scellement parce que l'expérience montre qu'elle est souvent moins résistante que l'interface scellement-terrain, en particulier dans le domaine des milieux rocheux (Hyett et al., 1995 ; Kaiser et al., 1992 ; Moosavi et al., 2005 ; Pakalnis et al., 1994 ; Reichert et al., 1992 ; Stillborg, 1986).

L'interface barre-scellement est définie comme la surface de contact entre le boulon et le matériau de scellement. Elle est placée au rayon du boulon et sa forme épouse celle du profil de la barre. Afin d'étudier le comportement de l'interface, on doit créer un déplacement relatif entre la barre et le matériau de scellement, ce qui produit une décohésion entre les deux matériaux. Dans la pratique, ceci est souvent effectué moyennant l'essai d'arrachement, qui consiste à appliquer un effort normal de traction sur une extrémité d'une tige ancrée, l'autre extrémité étant libre (force nulle). Au fur et à mesure que la décohésion se produit, un joint se développe entre la barre et le matériau de scellement. La décohésion est un phénomène local qui se propage vers l'extrémité libre (ou non chargée) de la barre avec l'endommagement de l'interface (Ivanovic et Neilson, 2009 ; Li et Stillborg, 1999 ; Ren et al., 2010).

Le but principal de cette recherche est de proposer un modèle de comportement pour l'interface barre-scellement. Ce modèle dépend de la combinaison barre-scellement choisie. Comme dans le cas des joints rocheux, le comportement de l'interface est défini dans les directions normale et tangentielle (l'une des directions tangentielles correspondant à la direction axiale de la barre). Le comportement de l'interface a un rôle clé dans le transfert de charge entre le soutènement et le terrain et, par extension, dans la réponse du boulonnage à ancrage réparti. Par ailleurs, la loi de comportement de l'interface peut être implémentée dans des logiciels de simulation numérique, ce qui permet de faire de meilleures prédictions sur le comportement des ouvrages renforcés par ce type de soutènement. Plusieurs études ont montré que la réponse du boulonnage à ancrage réparti peut être décrite numériquement (Laigle, 2004 ; Launay, 2010), d'où l'importance de bien modéliser tous ses éléments, y compris les interfaces.

La revue bibliographique montre que la plupart des études se sont focalisées uniquement sur la réponse tangentielle à l'interface. Une des premières études publiées est celle de Farmer (1975), qui a développé une solution analytique pour la distribution axiale de la contrainte de cisaillement lorsqu'un boulon à ancrage réparti est soumis à un effort axial de traction.
Dans cette étude, Farmer a supposé que la roche est infiniment rigide et n'a pas considéré le phénomène de décohésion de l'interface, ce qui mène à une décroissance exponentielle de la contrainte de cisaillement avec la distance au point d'application de l'effort. Postérieurement, Li et Stillborg (1999) ont complété l'étude de Farmer en tenant compte de l'élasticité du terrain dans la réponse de l'interface et en incluant le phénomène de décohésion. La notion de front de décohésion a été ainsi introduite ; ce front se propage vers l'extrémité non chargée de la barre avec l'endommagement de l'interface. D'après Li et Stillborg (1999), la contrainte de cisaillement est constante lorsque l'interface est en phase résiduelle (à proximité de l'extrémité chargée), et augmente de façon linéaire jusqu'au front de décohésion. Au-delà, le cisaillement décroît de façon exponentielle jusqu'à l'extrémité libre de la barre (i.e., pas de décohésion), de façon similaire au modèle proposé par Farmer (1975).

Benmokrane et al. (1995) ont proposé un modèle trilinéaire entre la contrainte de cisaillement et le glissement tangentiel à l'interface. La première partie du modèle, à pente positive, représente l'élasticité de l'interface; la deuxième partie, à pente négative, en représente l'endommagement et la dernière partie, à palier constant, tient compte d'une résistance résiduelle due au frottement. Récemment, Ren et al. (2010) ont développé un outil analytique permettant de retrouver le modèle tri-linéaire d'interface qui ajuste au mieux les résultats d'un essai d'arrachement (force axiale en fonction du glissement). Cet outil est basé sur cinq stades de la distribution axiale de la contrainte de cisaillement (d'un stade où toute la longueur ancrée est en phase élastique à un stade où l'interface est totalement en phase résiduelle); ces phases apparaissent à l'extrémité de la barre soumise au chargement et se propagent vers l'extrémité libre (i.e., non chargée) au fur et à mesure que le glissement augmente.

Concernant les câbles d'ancrage, Yacizi et Kaiser (1992) ont proposé le Bond Strength Model (BSM). Ce modèle conceptuel explique la rupture à l'interface d'un système de soutènement par câblage. Il tient compte des contributions tangentielle et normale, mais n'est utile que pour déterminer la résistance ultime du renforcement, car le processus d'endommagement de l'interface n'est pas considéré. Par ailleurs, la validité de ce modèle empirique est limitée aux cas de faibles longueurs d'ancrage.

Hyett et al. (1995) ont proposé une loi d'interface complète, incluant les réponses normale et tangentielle à l'interface, pour des câbles standard à 7 torons scellés au coulis de ciment. Cette loi d'interface est basée sur des aspects théoriques et empiriques. Le comportement tangentiel à l'interface est purement frictionnel, avec une relation de proportionnalité directe entre la pression normale à l'interface et la contrainte de cisaillement. Le comportement normal à l'interface est inspiré de celui des joints rocheux (Saeb et Amadei, 1992), et est modélisé comme un joint non linéaire avec dilatance. La comparaison, dans les directions normale et tangentielle, entre les prédictions obtenues par cette loi et les résultats des essais d'arrachement est assez satisfaisante. Néanmoins, cette loi repose sur des hypothèses discutables du comportement de l'interface : comportement normal supposé similaire à celui des joints rocheux et comportement axial purement frictionnel. Par ailleurs, l'applicabilité de cette loi à des boulons à ancrage réparti n'a pas été investiguée dans les publications. 
En résumé, le comportement de l'interface barre-scellement $\mathrm{du}$ boulonnage à ancrage réparti n'est pas complètement maîtrisé à l'heure actuelle. Nous proposons ici d'améliorer les connaissances sur cette interface, dans l'objectif à long terme de déterminer sa loi de comportement. Nous ne faisons aucune hypothèse sur la réponse de l'interface, et considérons des conditions statiques. Par ailleurs, en raison des conditions habituelles in situ, seules des sollicitations monotones sont prises en compte.

Dans cet article, nous présentons d'abord les aspects fondamentaux des outils analytiques permettant d'accéder au comportement de l'interface à partir des données mesurables pendant un essai d'arrachement. Ensuite, nous décrivons les études expérimentales effectuées en laboratoire et in situ pour améliorer les connaissances actuelles sur la réponse de l'interface. Après, nous expliquons la nouvelle méthode développée pour déterminer les réponses normale et tangentielle de l'interface barre-scellement, en combinant les outils analytiques et les résultats expérimentaux. Finalement, nous présentons un exemple d'application de cette nouvelle méthode.

\section{Outils analytiques pour l'étude de l'interface barre-scellement}

\subsection{Définitions préliminaires}

La réponse de l'interface barre-scellement est définie par quatre variables : le glissement axial, $W$, la contrainte de cisaillement, $\tau_{b}$, la pression normale à l'interface, $p_{b}$, et l'ouverture radiale, $\Delta u_{r b}$, donnée par:

$$
\Delta u_{r b}=u_{r b^{+}}-u_{r b^{-}}
$$

où $u_{r b^{+}}$et $u_{r b^{-}}$sont respectivement les déplacements radiaux du scellement et de la barre. L'indice $b$ désigne l'interface barrescellement, placée au rayon $r=R_{b}$. La Figure 1 montre un schéma de l'interface, ainsi que ces quatre variables. Pour des raisons géométriques, un système de référence cylindrique est utilisé. L'axe $Z$ correspond à l'axe de la barre. Comme le montre la figure, le glissement et le cisaillement sont définis dans la direction axiale ou tangentielle à l'interface, tandis que la pression et l'ouverture radiale sont définies dans la direction radiale ou normale à l'interface.

Lorsque la variable de contrôle est le déplacement axial (situation la plus courante in situ), le glissement est imposé et la pression normale est donnée par les conditions aux limites. Ainsi, les variables indépendantes sont $W$ et $p_{b}$, et la réponse de l'interface barre-scellement est décrite par les expressions $\tau_{b}\left(W, p_{b}\right)$ et $\Delta u_{r b}\left(W, p_{b}\right)$. Ces deux expressions constituent la loi de comportement de l'interface barre-scellement.

Du fait de sa localisation, l'interface barre-scellement ne peut pas être étudiée directement. En effet, comme le montre la Figure 1, la seule variable d'interface qui peut être mesurée pendant un essai d'arrachement est le glissement $W$ (obtenu en retranchant au déplacement axial imposé, $U_{a}$, le déplacement dû à l'élasticité de la longueur non ancrée de la barre [on suppose que le scellement et le terrain sont bloqués dans la direction axiale]). Pour cette raison, des mesures sont prises dans les matériaux environnants et converties pendant l'analyse des résultats en variables d'interface. Les matériaux environnants sont la barre et le scellement. Bien que le terrain environnant ne soit pas indispensable pour l'étude de l'interface barre-scellement, il est souvent pris en compte dans les essais d'arrachement en laboratoire, afin de pouvoir étudier les deux interfaces.

Typiquement, les variables mesurées pendant un essai d'arrachement effectué en laboratoire sont : le déplacement axial à l'extrémité chargée, $U_{a}$, la pression radiale à l'extérieur de l'échantillon, $p_{r}$, la force axiale appliquée sur la barre, $F$, et, dans certaines configurations, le déplacement radial de l'échantillon, $u_{r r}$ ( $c f$. Fig. 1). La force $F$ et le déplacement $U_{a}$ sont définis dans la direction axiale, et la pression $p_{r}$ et le déplacement $u_{r r}$ sont définis dans la direction radiale. Finalement, on remarquera que dans un essai effectué in situ, les seules variables mesurables sont $U_{a}$ et $F$. De ce fait, les essais en laboratoire sont préférables pour l'étude de l'interface. C'est dans ce cadre que l'on se place ici pour étudier les réponses normale et tangentielle de l'interface.

Dans la configuration montrée dans la Figure 1, l'équilibre de la barre est donné par :

$$
d F=2 \pi R_{b} \tau_{b} d Z
$$

En outre, l'équilibre des matériaux environnants, dans un système de référence cylindrique, s'écrit :

$$
\begin{gathered}
\frac{\partial \sigma_{r r}}{\partial r}+\frac{\partial \sigma_{r Z}}{\partial Z}+\frac{\sigma_{r r}-\sigma_{\theta \theta}}{r}=0 \\
\frac{\partial \sigma_{Z Z}}{\partial Z}+\frac{\partial \sigma_{r Z}}{\partial r}+\frac{\sigma_{r Z}}{r}=0
\end{gathered}
$$

où $\sigma_{r Z}=\tau_{b}$ pour $r=R_{b}$.

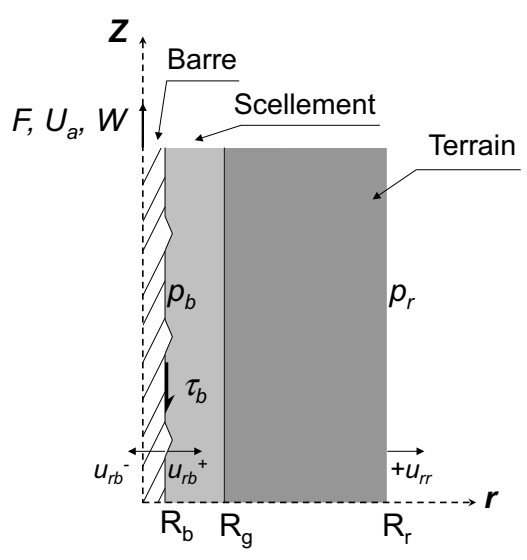

Fig. 1. Vue schématique de l'interface barre-scellement $\left(r=R_{b}\right)$ et des matériaux environnants, avec positionnement des variables d'interface et variables mesurables. Les rayons extérieurs du scellement et du terrain sont $R_{g}$ et $R_{r}$ respectivement.

Fig. 1. Schematic representation of the bolt-grout interface $\left(r=R_{b}\right)$ and the surrounding materials. The interface and observable variables are located. The borehole and rock sample outer radii are denoted by $R_{g}$ and $R_{r}$ respectively. 
Ces équations, qui correspondent à un problème de révolution, sont ensuite simplifiées en suivant Popov (1978) et ce en négligeant les termes dérivés par rapport à $Z$. Cette simplification permet de dissocier les directions radiale et axiale et de mener deux études unidimensionnelles, présentées ci-dessous.

\subsection{Réponse normale à l'interface}

La combinaison de l'équation (3) avec les équations décrivant le comportement de l'anneau de scellement et de terrain permet de déterminer $p_{b}$ et $u_{r b^{+}}$en fonction de $p_{r}$ et $u_{r r}$. De façon similaire, l'équation (3) et la rhéologie de la barre permettent de déterminer $u_{r b^{-}}$(développements analytiques très classiques, $c f$. Popov, 1978). L'ouverture radiale peut ainsi être déterminée grâce à l'équation (1). Plus de détails seront donnés dans la Section 4.

Dans cette étude, la réponse des matériaux environnants a été supposée élastique, ce qui mène à des développements analytiques aisés (Blanco-Martín, 2012). Les essais d'arrachement ont été effectués en veillant à vérifier cette hypothèse. En effet, l'étude de l'interface est plus directe si les matériaux environnants suivent un comportement élastique. $\mathrm{Si}$, par exemple, l'anneau de scellement ou de terrain se fracture radialement, la rigidité radiale change pendant l'essai et l'interprétation des résultats et la détermination des variables d'interface deviennent plus compliquées. De la même façon, l'analyse des résultats est plus simple si la barre d'ancrage ne plastifie pas.

Lorsque le déplacement $u_{r r}$ n'est pas mesuré directement, il peut être déterminé en utilisant la variation volumique du fluide confinant l'échantillon en laboratoire, grâce à l'expression :

$$
\frac{\Delta V}{V_{0}}=\frac{2 \pi H}{V_{0}}\left(R_{c} \Delta u_{r c}-R_{r} \Delta u_{r r}\right)
$$

où $\Delta V=V-V_{O}$ est la variation volumique du fluide confinant, $V_{O}$ est le volume initial du fluide, $H$ est la hauteur de l'échantillon, $R_{c}$ est le rayon intérieur de la cellule de confinement et $u_{r c}$ est le déplacement radial de la cellule à $r=R_{c}$.

\subsection{Réponse tangentielle à l'interface}

La combinaison de l'équation (4) avec le comportement de l'anneau de scellement et de terrain permet de déterminer le cisaillement $\tau_{b}$ avant la décohésion de l'interface, i.e., lorsqu'il y a continuité du déplacement axial (Blanco-Martín, 2012). Ceci limite l'intérêt de la réponse tangentielle des anneaux dans le cadre de l'étude de l'interface barre-scellement.

Pour pallier cette limitation, un nouvel outil analytique a été développé (Blanco-Martín et al., 2011a). Cet outil fait le lien entre la réponse de l'interface, $\tau_{b}(W)$, et une courbe de charge expérimentale, $F(W)$. En particulier, il permet de déterminer la relation $\tau_{b}(W)$ qui prédit au mieux une courbe $F(W)$. Par rapport aux outils analytiques décrits dans la littérature, ce nouvel outil n'est pas limité à un nombre donné de stades de la distribution axiale de la contrainte de cisaillement le long de la partie ancrée, $\tau_{b}(Z)$, et peut être utilisé lorsque la longueur d'ancrage diminue ou reste constante pendant l'arrachement. Par ailleurs, il est valable pour tout modèle $\tau_{b}(W)$, et non seulement pour des modèles tri-linéaires. D'autre part, ses limitations sont celles liées au cadre général de cette recherche: sollicitations monotones et barre en régime élastique.

L'utilisation de ce nouvel outil pour prédire la réponse de plusieurs essais d'arrachement dont la seule différence est la longueur initiale d'ancrage montre clairement que le lien entre la force (mesurable) et le cisaillement (variable d'interface non mesurable) dépend de la longueur d'ancrage (Blanco-Martín et al., 2013) : lorsque la longueur $(L)$ est faible, $\tau_{b}$ est uniforme sur $Z$ et, en accord avec l'équation (2), $F$ et $\tau_{b}$ sont proportionnels. En revanche, pour de grandes longueurs d'ancrage, $\tau_{b}(Z)$ n'est pas uniforme sur $L$ et la conversion $F-\tau_{b}$ doit être effectuée à l'aide d'un outil analytique. La Figure 2 illustre l'effet de la longueur d'ancrage sur la réponse à l'arrachement.

\subsection{Conclusions}

Les outils analytiques permettent d'obtenir les variables d'interface non mesurables : $\tau_{b}, p_{b}$ et $\Delta u_{r b}$. D'autre part, la loi d'interface barre-scellement est donnée par les relations $\tau_{b}\left(W, p_{b}\right)$ et $\Delta u_{r b}\left(W, p_{b}\right)$. Avec un nombre suffisant d'essais, l'analyse des variables d'interface permet de déterminer ces relations. Cette analyse sera abordée dans la Section 4.

\section{Méthodes expérimentales et résultats}

\subsection{Nouveau banc d'essais à l'échelle du laboratoire}

Un nouveau banc d'arrachement a été conçu et mis en opération au Centre de Géosciences MINES-ParisTech (Blanco-Martín et al., 2013). Bien que le principe de fonctionnement de ce banc repose en partie sur des bancs existants (Hagan, 2004 ; Offner et al., 2000 ; Reynolds, 2006), sa conception est suffisamment flexible pour pouvoir tester un grand nombre de paramètres. En particulier, du fait de la présence du terrain environnant, il permet l'étude de la décohésion aux deux interfaces : barre-scellement et scellement-terrain. La rupture de la barre par dépassement de sa résistance à la traction est aussi possible.

La Figure 3 montre une coupe longitudinale du banc d'arrachement. Ce banc a deux parties différentes, la barre

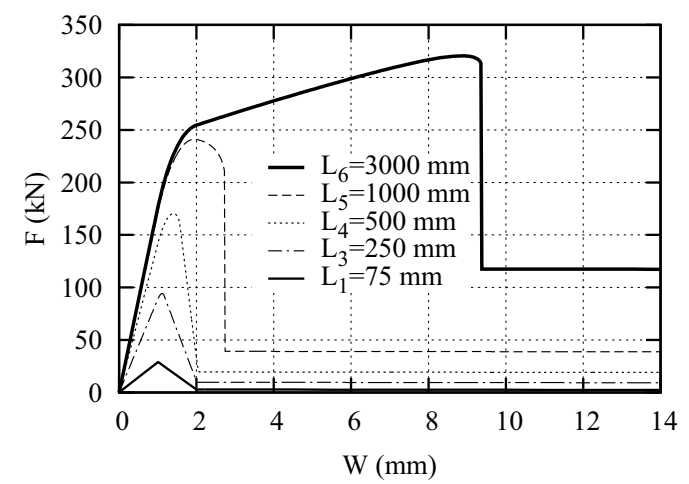

Fig. 2. Courbes de charge montrant l'effet de la longueur d'ancrage sur la réponse à l'arrachement.

Fig. 2. Load-displacement curves showing the effect of the embedment length on the pull-out response. 


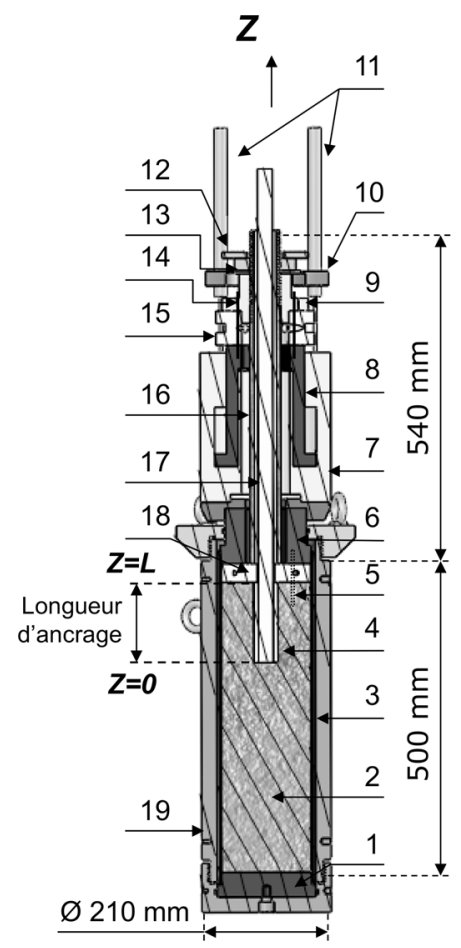

(1) Piston inférieur ;

(2) Échantillon de roche ;

(3) Jaquette ;

(4) Scellement;

(5) Goupilles pour empêcher la rotation;

(6) Piston supérieur ;

(7) Vérin hydraulique ;

(8) Piston du vérin ;

(9) Plaque filetée ;

(10) Pont capteurs LVDT ;

(11) Capteurs LVDT :

(12) Anneau de sécurité ;

(13) Contre-écrou ;

(14) Goupilles pour empêcher la rotation:

(15) Capteur de force ;

(16) Tube métallique ;

(17) Tige ;

(18) Plaque (effets de bord) ;

(19) Cellule biaxiale

Fig. 3. Coupe longitudinale du banc d'arrachement utilisé en laboratoire.

Fig. 3. Cutaway section of the laboratory pull-out test bench.

d'ancrage faisant le lien entre les deux. Dans la partie inférieure, la barre est scellée dans un trou borgne foré au préalable dans un échantillon cylindrique (roche, sol, ou matériau synthétique). L'échantillon est placé dans une cellule biaxiale qui permet d'appliquer une pression de confinement. Afin d'assurer une distribution uniforme du confinement et pour éviter les pressions de pore, l'échantillon est pourvu d'une jaquette (chemise en fluoro-élastomère). Par conception, la pression de confinement maximale est de $25 \mathrm{MPa}$.

Dans la partie supérieure, un tube métallique est ancré sur la longueur de la barre qui dépasse du trou borgne. Une résine à faible temps de prise est utilisée pour effectuer cet ancrage. On remarque que la longueur de barre ancrée dans le trou borgne est notamment inférieure à la longueur scellée dans le tube métallique, ce qui favorise la décohésion de l'interface à l'intérieur de l'échantillon, l'ancrage à l'intérieur du tube restant assuré. En effet, le tube métallique n'est utile que pour la mise en place des pièces nécessaires à l'arrachement. La qualité de l'ancrage supérieur a été testée moyennant un étalonnage du banc (Blanco-Martín, 2012).

Le tube métallique est fileté sur $180 \mathrm{~mm}$ à partir de son extrémité supérieure. Un vérin hydraulique repose sur le piston supérieur de la cellule biaxiale et sert à provoquer l'arrachement de la barre à l'intérieur de l'échantillon. Sur le piston (mobile) du vérin, on pose un capteur de force pourvu d'une plaque d'appui et de répartition. Le capteur de force a été spécifiquement conçu pour ce banc. Une plaque filetée est ensuite vissée sur le tube métallique jusqu'au contact avec le capteur de force. Sur cette plaque, on pose un pont pour les capteurs de déplacement. Ce pont est fixé par un écrou vissé sur
Tableau 1. Dimensions et limites de fonctionnement du nouveau banc d'arrachement.

Table 1. Dimensions and operational limits of the new pull-out bench.

\begin{tabular}{lll}
\hline Échantillon cylindrique & Longueur & $470 \pm 1 \mathrm{~mm}$ \\
& Diamètre extérieur & $144 \pm 1 \mathrm{~mm}$ \\
Jaquette & Hauteur & $560 \pm 1 \mathrm{~mm}$ \\
& Diamètre intérieur & $145 \pm 1 \mathrm{~mm}$ \\
& Épaisseur & $4 \pm 0,1 \mathrm{~mm}$ \\
Cellule biaxiale & Hauteur & $617 \pm 1 \mathrm{~mm}$ \\
& Diamètre intérieur & $160 \pm 1 \mathrm{~mm}$ \\
& Diamètre extérieur & $210 \pm 1 \mathrm{~mm}$ \\
& Pression maximale & $25 \mathrm{MPa}$ \\
Vérin hydraulique & Course maximale & $76 \mathrm{~mm}$ \\
& Force maximale & $931 \mathrm{kN}$ \\
Tube métallique & Poids & $63 \mathrm{~kg}$ \\
& Longueur & $540 \pm 5 \mathrm{~mm}$ \\
& Diamètre extérieur & $50 \pm 1 \mathrm{~mm}$ \\
Capteur de force & Diamètre intérieur & Variable \\
& Hauteur & $55 \mathrm{~mm}$ \\
Capteurs LVDT & Force maximale & $350 \mathrm{kN}$ \\
Capteurs de pression & Course maximale & $100 \mathrm{~mm}$ \\
& Pression maximale & $35 \mathrm{MPa}$ \\
\hline
\end{tabular}

le tube métallique. Le Tableau 1 montre les principales caractéristiques techniques du banc d'arrachement.

Dans l'espace entre les ancrages supérieur et inférieur $(3 \mathrm{~cm})$, entre l'échantillon et le piston supérieur de la cellule, on place une plaque métallique servant à assurer que la décohésion se produise à l'interface barre-scellement. Le diamètre du trou central de la plaque est donc celui de la barre à tester. Si l'on s'intéressait à l'interface scellement-terrain, le diamètre du trou de la plaque devrait être celui du trou borgne. Par ailleurs, dans le cas des milieux rocheux, la décohésion à l'interface scellement-terrain peut être facilitée par une faible rugosité du trou borgne.

Les goupilles entre l'échantillon et le piston supérieur de la cellule, et entre le piston du vérin et la plaque filetée, sont nécessaires dans le cas des câbles. En effet, du fait de leur faible résistance à la torsion, les câbles ont une tendance à se dévisser lorsqu'ils sont soumis à un effort axial de traction, en particulier lorsque la longueur d'ancrage est faible. Ce phénomène, bien connu à l'échelle du laboratoire et abordé dans des études préalables (Bawden et al., 1992), a peu d'importance pour les situations in situ, dans lesquelles les câbles ne peuvent pas se dévisser (à la place, un déroulement des torons pourrait avoir lieu, dû à la force axiale sur le câble qui favorise le redressement des torons). Par ailleurs, des études effectuées en laboratoire montrent que le dévissage des câbles fausse les résultats des essais d'arrachement. Afin d'éviter ce phénomène dans le nouveau banc, les goupilles ont été introduites pour bloquer toute rotation relative entre les composants des parties supérieure et inférieure du banc. À ce stade, seule la rotation relative entre le corps du vérin et son piston est possible ; pour l'empêcher, un système a été conçu et son efficacité a été vérifiée (Blanco-Martín, 2012).

Lorsque le vérin est mis en opération, son piston se déplace verticalement vers le haut, ce qui provoque l'arrachement dans la longueur d'ancrage, à l'intérieur du trou borgne. Comme le montre la Figure 3, la réaction du vérin se fait sur l'échantillon 
cylindrique. La plaque métallique placée sur l'échantillon permet de mieux distribuer cette réaction. Alternativement, la réaction du vérin pourrait être transférée soit à la facette inférieure de l'échantillon, soit sur une surface d'appui (la cellule étant fixée alors par un autre moyen). Cependant, ces configurations alternatives sont très compliquées à mettre en œuvre (Hagan, 2004).

L'essai est effectué en conditions statiques, à vitesse de déplacement contrôlée $(0,02 \mathrm{~mm} / \mathrm{s})$. Les variables mesurées pendant l'essai sont : la pression de confinement, la force axiale et le déplacement axial. La fréquence d'acquisition est de $5 \mathrm{~Hz}$. Le déplacement axial est mesuré moyennant trois capteurs LVDT, fixés dans le pont montré dans la Figure 3. Ainsi, le déplacement mesuré correspond à la somme du déplacement axial de la barre au point $Z=L$ et de la variation de sa longueur libre entre les ancrages supérieur et inférieur. On remarque que la longueur d'ancrage diminue pendant l'essai. Les avantages d'une longueur d'ancrage décroissante ont été soulignés par Verderame et al. (2009).

Par ailleurs, les essais peuvent être effectués soit à rigidité radiale constante, soit à pression de confinement constante. Dans le premier cas, la cellule de confinement est un système fermé et la pression du fluide confinant peut varier pendant l'arrachement. Dans le deuxième cas, la pression de confinement est maintenue constante pendant l'essai (i.e., système non fermé).

Les paramètres qui peuvent être étudiés à l'aide de ce banc sont : la longueur d'ancrage, la pression de confinement, le diamètre du trou borgne, la rugosité de ce trou, et le type de barre, de scellement et de terrain employés.

À l'exception du filetage des tubes métalliques, la préparation des échantillons est effectuée au Centre de Géosciences MINES-ParisTech, suivant des procédures dédiées (Blanco-Martín, 2012).

\subsection{Campagne expérimentale et résultats typiques}

Des essais d'arrachement ont été effectués sur des boulons et sur des câbles à ancrage réparti. Au total, 63 essais ont été effectués en laboratoire à l'aide du nouveau banc. Les essais ont été prolongés jusqu'à un glissement de 60-70 mm pour étudier la phase résiduelle.

Des boulons en acier crénelés HA25, des boulons en fibres de verre et des tiges en acier lisses ont été testés. Tous ces boulons ont un diamètre nominal de $25 \mathrm{~mm}$. La résistance des boulons HA25 est de $620 \mathrm{MPa}$, celle des boulons en fibres de verre est de $1060 \mathrm{MPa}$ et celle des tiges lisses est de $600 \mathrm{MPa}$. Comme matériau de scellement, on a utilisé une résine minière à prise lente (environ 3 minutes) et trois types de coulis de ciment, avec différents ratios eau-ciment, $e: c=\{0,3,0,35,0,4\}$ (Blanco-Martín et al., 2011b, 2013).

Parallèlement, des câbles de type Flexible de $23 \mathrm{~mm}$ de diamètre et à 7 torons ont été testés (1200 $\mathrm{MPa}$ de résistance). Le scellement a été effectué à la résine, car ce scellement est souvent employé in situ pour ce type de câble.

La plupart des essais ont été effectués en utilisant un échantillon en grès des Vosges. Des échantillons en granite ont été employés dans quelques essais. Le Tableau 2 montre les principales propriétés mécaniques des roches et des matériaux de scellement employés dans la plupart des essais.
Tableau 2. Propriétés mécaniques des roches et des matériaux de scellement utilisés pour la préparation de la plupart des échantillons. Table 2. Mechanical properties of the rocks and grouting materials used to prepare the majority of the samples.

\begin{tabular}{|c|c|c|c|}
\hline & $\begin{array}{l}\text { Grès des } \\
\text { Vosges }\end{array}$ & Résine & $\begin{array}{l}\text { Ciment } \\
(e: c=0,35)\end{array}$ \\
\hline Densité $\left(\mathrm{kg} / \mathrm{m}^{3}\right)$ & 2130 & 1987 & 1958 \\
\hline Module de Young (MPa) & 25600 & 11450 & 17500 \\
\hline Coefficient de Poisson (-) & 0,26 & 0,31 & 0,27 \\
\hline $\begin{array}{l}\text { Résistance à la compression } \\
\text { simple (MPa) }\end{array}$ & 62,4 & 67,1 & 44,1 \\
\hline Résistance à la traction $(\mathrm{MPa})$ & 3,1 & 12,1 & 3,2 \\
\hline Cohésion (MPa) & 12,4 & 20,2 & $*$ \\
\hline Angle de frottement $\left({ }^{\circ}\right)$ & 46 & 28 & * \\
\hline
\end{tabular}

* : valeur non déterminée.

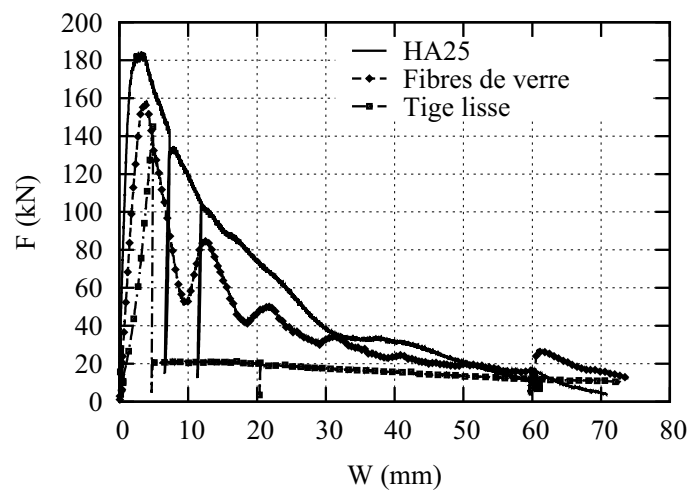

Fig. 4. Courbes de charge correspondantes à trois boulons différents. Fig. 4. Pull-out results for three different rockbolts.

Dans le cas des boulons, des longueurs faibles d'ancrage ont été retenues $(90-150 \mathrm{~mm})$, non seulement parce que l'analyse des résultats est plus simple ( $c f$. Sect. 2), mais aussi parce que la déduction de la relation cisaillement-glissement $\tau_{b}(W)$ est plus précise (Blanco-Martín et al., 2011b). Dans le cas des câbles, des longueurs d'ancrage entre 250 et $325 \mathrm{~mm}$ ont été employées : le but principal des essais d'arrachement effectués sur les câbles étant de vérifier l'efficacité du dispositif utilisé pour empêcher la rotation du banc, de grandes longueurs se sont avérées nécessaires. Finalement, les deux conditions aux limites, soit confinement constant, soit rigidité radiale constante, ont été utilisées.

La Figure 4 montre trois courbes de charge typiques, obtenues pendant trois essais effectués sous les mêmes conditions, mais sur trois boulons différents. La pression de confinement est de $5 \mathrm{MPa}$ (constante) et la longueur initiale d'ancrage est de $90 \mathrm{~mm}$.

Globalement, la courbe de charge peut être divisée en trois parties : avant le pic, la force augmente de façon quasi linéaire avec le glissement axial. Pendant cette phase, l'adhésion de l'interface, le frottement et l'éventuel blocage mécanique (dû au profil du boulon) contribuent à l'ancrage entre la barre et le scellement. Avant le pic, la réponse $F(W)$ devient non linéaire (bien que croissante encore) au fur et à mesure que l'endommagement de l'interface se développe. Un joint est 
progressivement créé entre la barre et le scellement. Dans la phase post-pic, l'endommagement graduel de l'interface (affaiblissement des contributions dues à l'adhésion et au blocage mécanique) réduit la force à une valeur résiduelle, due principalement au frottement.

Comme le montre la figure, le boulon en acier est le plus performant en termes de résistance à l'arrachement. Les principales différences entre les courbes de charge des trois barres ont lieu dans la phase post-pic. Dans le cas des tiges lisses, une fois l'adhésion disparue (au pic de force), la force diminue de façon soudaine jusqu'à une valeur résiduelle. En effet, la contribution due aux indentations (blocage mécanique) est absente dans ce type de barre, et seul le frottement reste après le pic de force. D'autre part, les boulons en fibres de verre présentent une phase post-pic caractérisée par une succession d'oscillations à amplitude décroissante. Ces oscillations sont dues au profil de la barre (leur périodicité est celle du profil du boulon) et leur amplitude diminue au fur et à mesure que l'endommagement de l'interface se développe. Finalement, le boulon HA25 présente une phase post-pic sans oscillation marquée. Ceci est dû à l'angle des indentations de ce type de boulon, plus fort que celui des barres en fibres de verre. En conséquence, lorsque le boulon est arraché, c'est le scellement entre les indentations qui est cisaillé, et l'interface est alors plus lisse.

Il est intéressant de remarquer que la force résiduelle des trois boulons tend vers la même valeur, une fois la contribution due au blocage mécanique minimisée (à cause de l'endommagement). De plus, cette force diminue progressivement de façon à ce que $F(W=L)=0$.

La Figure 5 montre les relations $\tau_{b}(W)$ de deux essais effectués sur des boulons HA25 et d'un essai effectué sur un câble Flexible. Étant donné que les longueurs d'ancrage utilisées dans ces essais sont différentes, la comparaison des résultats ne peut pas être effectuée en utilisant la force mesurée. Les essais ont été conduits à pression de confinement constante (indiquée dans la figure pour chaque essai).

Cette figure apporte deux informations intéressantes. Premièrement, la réponse post-pic des câbles est très différente de celle des boulons. La principale raison à ce constat est la différence des profils des deux barres, les câbles ayant des

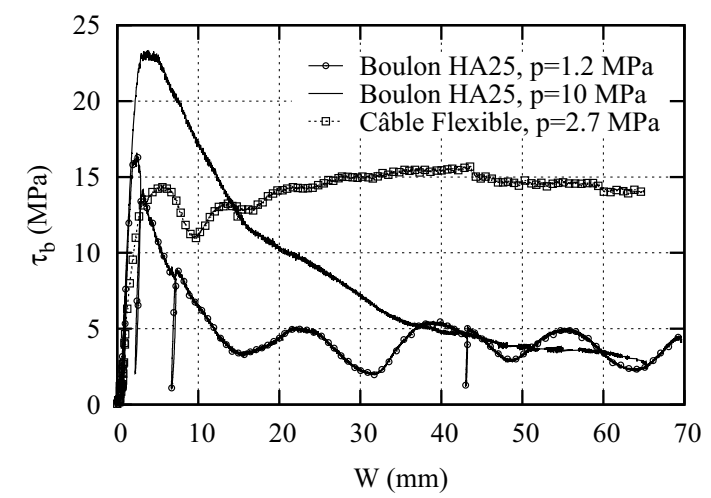

Fig. 5. Comparaison boulon-câble. Relations expérimentales $\tau_{b}(W)$ issues de trois essais d'arrachement.

Fig. 5. Comparison of pull-out test results on rockbolts and cablebolts. Experimental $\tau_{b}(W)$ curves from three tests. indentations beaucoup moins marquées que celles des boulons. Bien que la résistance maximum de l'ancrage ne présente pas de grande différence pour un même confinement, dans le cas des câbles le cisaillement résiduel est très similaire au cisaillement maximum. Compte tenu de ce résultat, l'emploi des câbles s'avère plus intéressant que celui des boulons lorsque l'on s'attend à avoir des glissements importants in situ.

Deuxièmement, pour un même type de barre, plus le confinement est fort, plus la contrainte de cisaillement développée à l'interface est élevée. Ceci est vrai pour toutes les barres testées, sous les deux conditions employées (confinement ou rigidité radiale constants). Par ailleurs, pour de forts confinements, l'endommagement de l'interface est plus accentué, ce qui lisse davantage l'interface. Ceci est corroboré par l'inspection visuelle des échantillons après les essais. En conséquence, les oscillations retrouvées dans la phase post-pic, et dues au profil des barres, sont d'autant moins marquées que le confinement est fort. D'autre part, pour de faibles confinements, l'anneau de scellement et de roche est susceptible de se fracturer radialement (Hyett et al., 1995 ; Yacizi et Kaiser, 1992). Dans cette situation, l'étude de l'interface s'avère compliquée pour les raisons suivantes : les blocs constituant l'anneau risquent d'être écartés radialement au fur et à mesure que la barre est arrachée, gênant le contact à l'interface ; comme expliqué dans la Section 2, l'analyse des résultats expérimentaux est plus complexe en cas de fracturation, puisque la rigidité radiale de l'anneau change (de plus, l'étude de la fracturation et de la propagation des fractures est au-delà de la portée de cette recherche). Pour ces raisons, il est préférable de contrôler l'exécution des essais pour empêcher l'occurrence des fractures dans l'anneau de scellement et dans le terrain. La Figure 6 montre l'aspect des anneaux après trois essais d'arrachement effectués sous les mêmes conditions, mais à trois confinements différents : $10 ; 5$ et $1,2 \mathrm{MPa}$.

\subsection{Essais in situ et comparaison laboratoire-in situ}

En 2009, l'Andra (Agence nationale pour la gestion des déchets radioactifs) a commandé une campagne d'essais d'arrachement dans le laboratoire de recherche souterrain de Bure/Haute-Marne. Afin de compléter cette campagne in situ avec une étude plus approfondie à échelle réduite en laboratoire, il a été décidé d'établir une collaboration entre l'Andra et le Centre de Géosciences MINES-ParisTech.

Soixante-six essais d'arrachement ont été effectués in situ sur quatre types de boulons : HA25, Swellex, Dywidag 26WR et $C T$-bolts. De la résine et un coulis de ciment (ratio $e: c=0,5$ ) ont été utilisés pour sceller les boulons Dywidag $26 W R$ et HA25. Les résultats obtenus ont été analysés (Blanco-Martín, 2012 ; Dénecé et al., 2009). Des longueurs d'ancrage caractéristiques des situations réelles ont été utilisées : $L=2,4$ et $3 \mathrm{~m}$. Afin d'éviter la plastification des barres, les essais ont été arrêtés à de faibles valeurs de déplacement axial $(\leq 5,5 \mathrm{~mm}$ pour les boulons HA25). La Figure 7 montre des résultats typiques obtenus pour les boulons $H A 25$; ces boulons ont été aussi testés à échelle réduite en laboratoire grâce au nouveau banc d'arrachement.

Bien que l'interface barre-scellement soit la même à grande et à petite échelles pour un même type de boulon et un même matériau de scellement (et donc, le modèle d'interface est le 

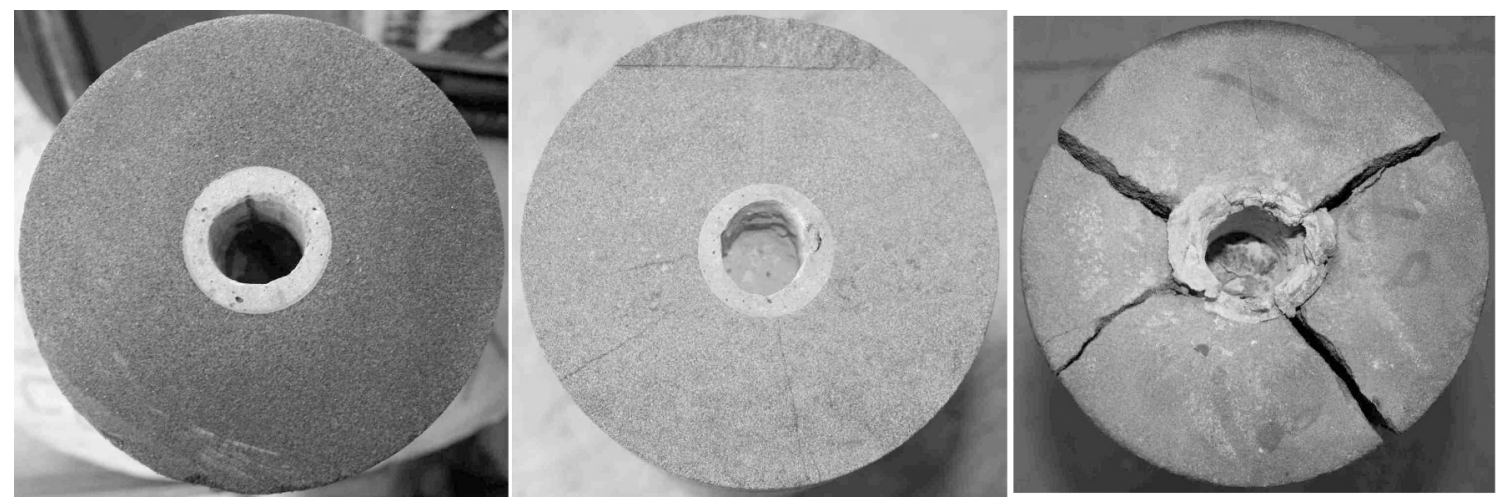

Fig. 6. Vue de l'anneau de scellement et de roche après trois essais effectués sur des boulons $H A 25$. De gauche à droite : $10 ; 5$ et 1,2 MPa. Fig. 6. Aspect of the grout and rock annulii after three pull-out tests conducted on HA25 rockbolts. From left to right: 10; 5 and 1.2 MPa.

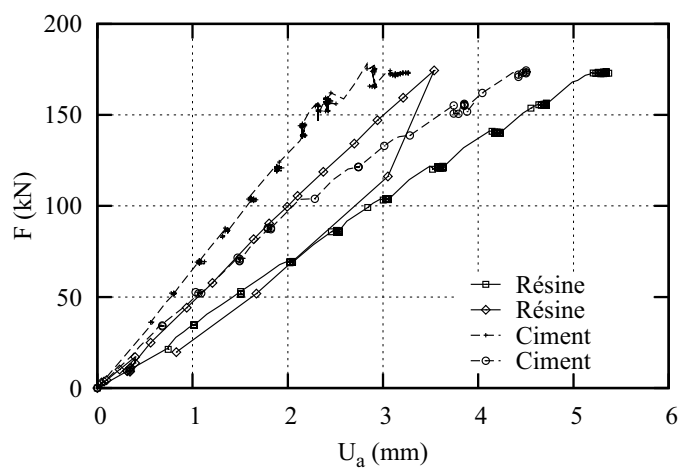

Fig. 7. Courbes de charge correspondantes à quatre essais d'arrachement effectués in situ sur des boulons HA25 (résultats bruts).

Fig. 7. Field pull-out test results on four HA25 rockbolts (raw data).

même), la réponse à l'arrachement sera différente pour plusieurs raisons. D'une part, la longueur d'ancrage utilisée est différente. Comme expliqué dans la Section 2, la réponse à l'arrachement (dont la courbe de charge $F(W)$ ) est fortement dépendante de la longueur d'ancrage ( $c f$. Fig. 2). D'autre part, les conditions aux limites sont différentes : au laboratoire, le banc d'arrachement permet d'effectuer les essais à confinement constant ou à rigidité radiale constante à l'extérieur de l'échantillon $\left(r=R_{r}\right)$, tandis que, in situ, les essais ont lieu à rigidité constante en paroi du trou borgne $\left(r=R_{g}\right)$. Cette rigidité est donnée par :

$$
K_{R g^{+}}=\frac{\partial p_{g}}{\partial u_{r g^{+}}}=\frac{E_{r}}{\left(1+v_{r}\right) R_{g}}
$$

où $E_{r}$ et $v_{r}$ sont les paramètres d'élasticité de la roche.

On voit ainsi que la réponse à l'arrachement sera différente dans les deux situations, même si le comportement de l'interface est décrit par les mêmes relations $\tau_{b}\left(W, p_{b}\right)$ et $\Delta u_{r b}\left(W, p_{b}\right)$.

En conclusion, la comparaison des résultats à l'arrachement obtenus in situ et en laboratoire, utile entre autres pour évaluer la validité des résultats obtenus à échelle réduite, n'est pas directe, et nécessite la modélisation des essais d'arrachement, une fois la loi de comportement de l'interface déterminée. Cette détermination s'avère plus facile et complète à l'échelle du laboratoire (grâce à davantage de mesures, notamment dans la direction radiale), lorsqu'on utilise de faibles longueurs d'ancrage.

\section{Formulation semi-empirique d'un modèle d'interface}

\subsection{Calcul des variables d'interface}

Comme annoncé précédemment, les variables mesurées au cours d'un essai en laboratoire sont la force axiale $F$, le déplacement axial $U_{\mathrm{a}}$, la pression de confinement $p_{r}$ et, parfois, le déplacement radial $u_{r r}$. Le glissement axial $W$ dans l'extrémité chargée est obtenu à partir du déplacement $U_{\mathrm{a}}$, en retranchant l'élasticité de la partie libre de la barre à l'extérieur du trou borgne ( $c f$. Fig. 3), et en appliquant la correction due à l'étalonnage du banc ( $c f$. Blanco-Martín (2012) pour une description détaillée de l'étalonnage).

Pour les faibles longueurs d'ancrage utilisées en laboratoire, le cisaillement $\tau_{b}$ peut être supposé constant sur $L$ (Blanco-Martín et al., 2013), ce qui permet de calculer facilement $\tau_{b}$ à partir de $F$. Pour une longueur d'ancrage décroissante, il vient :

$$
\tau_{b}=\frac{F}{2 \pi R_{b}(L-W)}
$$

Comme expliqué dans la Section 2, la pression à l'interface $p_{b}$ est déterminée à partir de $p_{r}$ et $u_{r r}$. Dans la présente étude, le déplacement $u_{r r}$ n'a pas été mesuré directement, mais calculé en combinant l'équation (5) avec l'équation d'état du fluide confinant. Cette équation ne peut être utilisée que lorsque la masse de fluide est constante. En conséquence, seuls les essais effectués à rigidité radiale constante ont été analysés dans un premier temps (ces essais représentent plus que la moitié des essais effectués en laboratoire). D'autre part, compte tenu que la longueur d'ancrage décroît au cours des essais effectués, la procédure analytique a été adaptée, de façon à ce que $p_{b}=0$ pour $Z \in[0, W]$. Cette procédure a été détaillée au préalable (Blanco-Martín, 2012). Il en résulte que :

$$
\begin{gathered}
\Delta p_{b}\left(1-\frac{W}{L}\right)=\frac{R_{j} \Delta p_{j}}{L \beta_{T} R_{b}}\left[\left(k+\frac{2 \pi R_{c}^{2} H \alpha_{c}}{V_{0}}\right) \frac{V_{0}}{2 \pi R_{j}^{2}}\right. \\
\left.+\gamma_{T} L+\gamma_{B}(H-L)\right]+p_{b}^{0} \frac{W}{L}
\end{gathered}
$$


où $p_{j}$ est la pression de confinement (appliquée sur la jaquette [indice $j]$ ), $R_{j}$ est le rayon extérieur de la jaquette, $k$ est la compressibilité du fluide confinant (supposée constante en dessous de $35 \mathrm{MPa}$ d'après les caractéristiques techniques (Enerpac, 2012)) et $\alpha_{c}, \beta_{T}, \gamma_{T}$ et $\gamma_{B}$ sont des constantes qui dépendent de la géométrie et des paramètres élastiques (Blanco-Martín, 2012). On remarque que la jaquette est prise en compte pour plus de précision dans le calcul de $p_{b}$.

Finalement, l'ouverture radiale $\Delta u_{r b}$ est déterminée moyennant :

$$
\begin{aligned}
\Delta u_{r b} & =u_{r b^{+}}-u_{r b^{-}} \\
& =\left(\alpha_{T}+\beta_{b}\right) R_{b} \Delta p_{b}-\beta_{T} R_{j} \Delta p_{j}
\end{aligned}
$$

où $\beta_{b}=\left(1-v_{b}\right) / E_{b}$. Le paramètre $\alpha_{T}$ est une constante, fonction de la géométrie et des paramètres élastiques du scellement, du terrain et de la jaquette.

Rappelons que la loi de comportement de l'interface est donnée par les expressions $\tau_{b}\left(W, p_{b}\right)$ et $\Delta u_{r b}\left(W, p_{b}\right)$. Ainsi, il est nécessaire de dissocier les contributions de $W$ et $p_{b}$ dans les équations (7) et (9). La comparaison des résultats expérimentaux dans les deux directions aide à cette dissociation. Un exemple d'application pour un boulon en fibres de verre est montré dans la section suivante.

\subsection{Exemple d'application}

La Figure 8 compare les relations $\tau_{b}(W)$ et $p_{j}(W)$ correspondantes à un essai d'arrachement effectué à rigidité radiale constante sur un boulon en fibres de verre. La pression de confinement initiale est de l'ordre de $5 \mathrm{MPa}$. Comme le montre la figure, l'allure des deux courbes est très similaire. Rappelons que $\tau_{b}$ est obtenu à partir de la réponse tangentielle à l'interface, et que $p_{j}$ (et par la suite $p_{b}$ d'après l'Éq. (8)) représente la réponse radiale. Malgré ces différences, les deux variables suivent une évolution très similaire. Par ailleurs, la pression commence à augmenter de façon significative pour $W \approx 0$, avant le premier pic de $\tau_{b}$. On peut remarquer dans la figure qu'il y a un déphasage entre le premier pic des deux courbes, tandis que le deuxième pic et les pics suivants sont

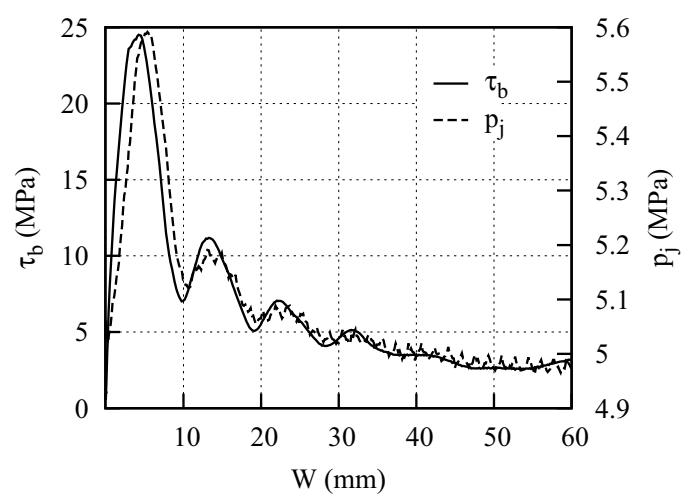

Fig. 8. Exemple d'application de la nouvelle méthode pour accéder au comportement de l'interface. Comparaison des courbes $\tau_{b}(W)$ et $p_{j}(W)$. Fig. 8. Example showing the application of the new method to access the interface behaviour. Comparison of curves $\tau_{b}(W)$ and $p_{j}(W)$. en phase. Finalement, l'amplitude des oscillations en phase post-pic décroît avec le glissement. Ces oscillations, dues au profil de la barre, se remarquent sur les deux courbes.

La cause la plus probable pour l'augmentation de la pression à $W \approx 0$ et pour le déphasage initial est la création d'un joint à $r=R_{b}$. Ce joint apparaît à $Z=L$ et se propage vers l'extrémité libre (i.e., non chargée) de la barre lorsque $W$ augmente. Avant le premier pic de $\tau_{b}$, l'interface présente encore ses trois composantes d'ancrage (adhésion, frottement et blocage mécanique), même si elle n'est pas totalement couplée, une fois le joint apparu à $Z=L$. Au pic de $\tau_{b}$, l'interface est dissociée et le joint complètement développé. À partir de ce point, la pression et le cisaillement sont proportionnels, confirmant l'hypothèse classique d'une relation linéaire entre ces deux variables. De ce fait, le frottement constitue la plus grande contribution à l'ancrage en phase post-pic.

Pour caractériser ces observations, nous proposons un modèle de cisaillement constitué de deux composantes. La première composante, $\tau_{c}$, définie à pression constante, tient compte de l'adhésion et du blocage mécanique. Cette composante s'exprime par l'égalité :

$\tau_{c}(W)= \begin{cases}\frac{\tau_{p}}{W_{p}} W & \text { pour } 0 \leq W \leq W_{p} \\ \tau_{r}+\left(\tau_{p}-\tau_{r}\right) & \left(\frac{W_{r}-W}{W_{r}-W_{p}}\right)^{2} \text { pour } W_{p} \leq W \leq W_{r} \\ \tau_{r} & \text { pour } W \geq W_{r}\end{cases}$

Les indices $p$ et $r$ se réfèrent au pic et à la phase résiduelle respectivement. Il s'agit donc d'un modèle à trois phases, similaire à celui proposé par Benmokrane et al. (1995); cependant, la deuxième phase est non linéaire pour mieux reproduire les résultats expérimentaux.

La seconde composante, $\tau_{v}$, est proportionnelle à la pression et représente l'effet du frottement. Elle reflète la ressemblance des courbes $\tau_{b}(W)$ et $p_{b}(W)$ après la décohésion de l'interface et s'exprime comme suit :

$$
\tau_{v}\left(\Delta p_{b}\right)=\Delta p_{b} \tan \varphi
$$

Ainsi, le cisaillement total obéit à la relation :

$$
\tau_{b}\left(W, \Delta p_{b}\right)=\tau_{c}(W)+\tau_{v}\left(\Delta p_{b}\right)
$$

Au total, cinq paramètres sont nécessaires pour définir $\tau_{b}$ : $W_{r}, W_{p}, \tau_{p}, \tau_{r}$ et $\varphi$. La Figure 9 montre la comparaison entre le cisaillement expérimental et le cisaillement modélisé avec les équations (10) à (12). Les composantes $\tau_{c}$ et $\tau_{v}$ sont aussi représentées pour montrer leurs contributions respectives. Comme le montre cette figure, la comparaison est assez satisfaisante.

Quant à l'ouverture radiale $\Delta u_{r b}$, la dissociation des contributions relatives de $W$ et $p_{b}$ est plus complexe. En effet, $\Delta u_{r b}$ est calculée directement à partir de $p_{b}$ grâce à l'équation (9), donc une comparaison $\Delta u_{r b}(W)$ et $p_{b}(W)$ (ou $p_{j}(W)$ ) s'avère moins utile que pour le cas du cisaillement. La réalisation d'un plus grand nombre d'essais permettrait de dissocier les contributions de $W$ et $p_{b}$. Pour le moment, il a été supposé 


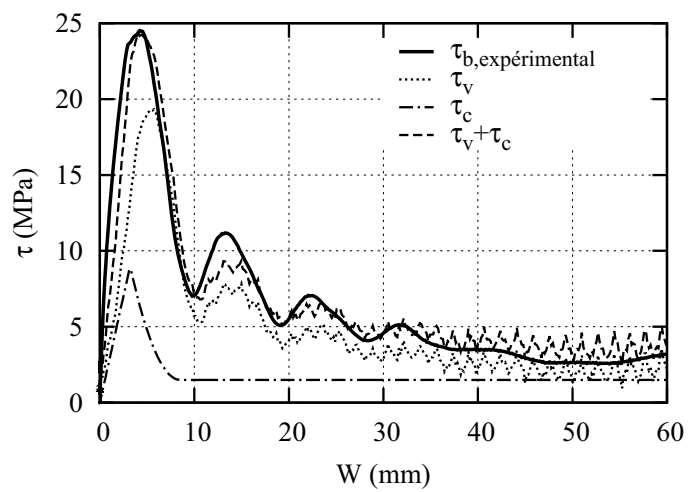

Fig. 9. Exemple d'application de la nouvelle méthode pour accéder au comportement de l'interface. Comparaison du cisaillement expérimental et modélisé (avec les contributions $\tau_{c}$ et $\tau_{v}$ ).

Fig. 9. Example showing the application of the new method to access the interface behaviour. Comparison between experimental and modelled shear stress (with the components $\tau_{c}$ and $\tau_{v}$ ).

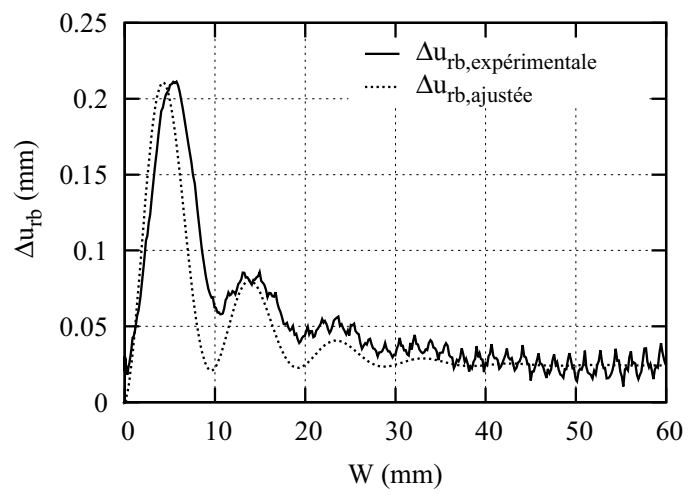

Fig. 10. Exemple d'application de la nouvelle méthode pour accéder au comportement de l'interface. Comparaison de l'ouverture radiale expérimentale et modélisée.

Fig. 10. Example showing the application of the new method to access the interface behaviour. Comparison between experimental and modelled radial opening.

que l'ouverture radiale ne dépend que de $W$. Pour les boulons en fibres de verre, on retient la relation :

$$
\begin{aligned}
\Delta u_{r b}(W) & =b \exp \left(-\frac{W}{c}\right)\left(1-\cos \left(2 \pi \frac{W}{a}\right)\right) \\
& +\frac{d_{f}}{1++^{a} / 5 W}
\end{aligned}
$$

Quatre paramètres sont nécessaires pour décrire cette ouverture : $b$ est l'amplitude initiale, $c$ est la distance d'arrachement caractéristique, et $d_{f}$ est l'ouverture résiduelle. La constante $a$ n'est pas un paramètre d'ajustement, mais une caractéristique géométrique (la périodicité du profil de la barre, i.e., la distance moyenne entre les indentations).

La Figure 10 montre une comparaison entre l'ouverture expérimentale et l'ouverture modélisée avec l'équation (13). Même si le modèle doit être complété, la comparaison est satisfaisante.

Il est important de remarquer que le modèle d'interface dépend de la combinaison barre-scellement choisie : de ce fait, le modèle décrit ici pour les boulons en fibres de verre scellés à la résine ne doit pas être considéré comme un modèle général d'interface. C'est la méthode d'accès à ce modèle qui est générale à toute interface barre-scellement.

\section{Conclusion générale}

La maîtrise du boulonnage à ancrage réparti - au sens large : boulonnage et câblage - nécessite une bonne compréhension du transfert de charge entre le renforcement et le terrain environnant. Dans ce contexte, le comportement des interfaces barre-scellement et scellement-terrain doit être bien compris. Dans cette étude, nous nous intéressons à l'interface barrescellement parce que l'expérience montre qu'elle est souvent moins résistante que l'interface scellement-terrain, en particulier dans le domaine des milieux rocheux. Afin d'étudier l'interface, un déplacement relatif entre la barre et le matériau de scellement doit être créé. Ceci est souvent effectué moyennant un effort axial de traction appliqué sur la barre, le reste des composants étant fixé.

La principale difficulté associée à l'étude de l'interface est son accès difficile, ce qui empêche la prise directe de mesures, à l'exception du déplacement axial. De ce fait, les mesures prises dans les matériaux environnants (barre, scellement, terrain) doivent être converties, moyennant des outils analytiques, en variables d'interface. Ainsi, la combinaison des résultats expérimentaux, obtenus à partir des essais d'arrachement effectués sous des conditions bien contrôlées, et des outils analytiques, faisant le lien entre variables observables et variable d'interface, permet d'accéder à la réponse de l'interface. Comme pour les joints rocheux, cette réponse est définie dans les directions tangentielle et normale. Par ailleurs, elle dépend de la combinaison barrescellement choisie. Pour cette raison, il est plus intéressant de développer une méthode générale d'accès à l'interface que de proposer un modèle particulier d'interface. Le développement de cette méthode constitue l'apport principal de ce travail.

Les essais d'arrachement effectués à petite échelle en laboratoire sont plus facilement contrôlables et permettent l'accès à plus de mesures que les essais in situ, notamment dans la direction normale à l'interface. Ainsi, il est préférable de mener l'étude expérimentale à petite échelle en laboratoire. Des outils analytiques sont ensuite employés pour l'analyse des résultats obtenus. Une fois la loi d'interface déterminée, la modélisation des essais à grande échelle et la comparaison avec des résultats expérimentaux permettront d'évaluer la recherche menée à de faibles longueurs d'ancrage. Un nouvel outil analytique permettant de prédire la réponse à l'arrachement d'un boulon ancré sur une grande longueur a été développé et validé. D'autre part, un nouveau banc d'arrachement a été conçu et progressivement amélioré de façon à étudier l'interface avec autant de précision que possible. Afin de faciliter l'analyse des résultats et de ne pas introduire des incertitudes non liées à l'interface, il est préférable que les matériaux environnants restent dans le domaine de l'élasticité.

À partir de la méthode proposée pour accéder à l'interface barre-scellement, des modèles préliminaires d'interface ont été développés pour quelques combinaisons barre-scellement. Ces modèles devront être parachevés lorsque nous disposerons de plus de résultats pour établir une loi d'interface complète. En particulier, des mesures plus précises dans la direction radiale 
sont nécessaires. Mais en tous cas, ils ont en commun une série d'aspects. Premièrement, le frottement n'est pas le seul composant du cisaillement, bien qu'il soit dominant en phase post-pic. Par ailleurs, l'endommagement de l'interface dépend non seulement du frottement (dû à la pression normale), mais aussi des caractéristiques géométriques du profil de la barre. Deuxièmement, le profil de la barre doit être pris en compte lorsque de grands glissements peuvent avoir lieu. Dans ce contexte, l'emploi de câbles semble être plus bénéfique (mais au pic, les boulons et les câbles testés montrent des performances similaires dans les mêmes conditions). Troisièmement, l'ouverture normale à l'interface dépend principalement du profil de la barre.

Une utilité importante du modèle d'interface est son implémentation dans un code de calcul, permettant de faire des prédictions plus précises du comportement des ouvrages complexes renforcés par des boulons et des câbles à ancrage réparti, et aussi de mieux dimensionner les schémas de soutènement par boulonnage. Ainsi, l'emploi d'un effort axial de traction pour étudier l'interface est justifié : ce sera le code de calcul qui fera la superposition des différents chargements auxquels le renforcement est soumis. L'aspect critique est alors la bonne compréhension de toutes les parties intégrantes du renforcement, interfaces incluses.

Remerciements. Le partenariat avec l'Andra, dans le cadre du projet Mibouland, a mis à disposition du Centre de Géosciences MINES-ParisTech une partie du matériel nécessaire à la réalisation des essais d'arrachement en laboratoire, et aussi les résultats des essais in situ. La participation dans le projet européen Prosafecoal a aussi contribué de façon significative à l'avancement de cette recherche. Un des objectifs de ce projet est d'améliorer la productivité et la sécurité des mines de charbon européennes moyennant l'utilisation du boulonnage à ancrage réparti dans les intersections entre le front d'exploitation et les voies d'accès.

\section{Références}

Bawden W, Hyett A, Lausch P. 1992. An experimental procedure for the testing of cable bolts in situ. Int J Rock Mech Min Sci 29: 525-533.

Benmokrane B, Chennouf A, Mitri HS. 1995. Laboratory evaluation of cementbased grouts and grouted rock anchors. Int J Rock Mech Min Sci 32: 633-642.

Blanco-Martín L. 2012. Theoretical and experimental study of fully grouted rockbolts and cablebolts under axial loads. Mémoire de thèse, MINES-ParisTech (France).

Blanco-Martín L, Tijani M, Hadj-Hassen F. 2011a. A new analytical solution to the mechanical behaviour of fully grouted rockbolts subjected to pull-out tests. Constr Build Mater 25: 749-755.

Blanco-Martín L, Hadj-Hassen F, Tijani M. 2011b. A new experimental and analytical study of fully grouted rockbolts. In: Proceedings of the 45th US Rock Mechanics/Geomechanics Symposium, San Francisco (USA).

Blanco-Martín L, Tijani M, Hadj-Hassen F, Noiret A. 2013. Assessment of the boltgrout interface behaviour of fully-grouted rockbolts from laboratory experiments under axial loads. Int J Rock Mech Min Sci 63: 50-61.
Dénecé D, Leleu A, Beth M. 2009. Essais de traction boulons d'ancrage. Campagne octobre 2009. Rapport des essais, Sol Data (pour l'Andra).

Enerpac Learning Center. Disponible sur http://www.enerpac.com/en/ basic-hydraulics (2012).

Farmer IW. 1975. Stress distribution along a resin grouted rock anchor. Int J Rock Mech Min Sci 12: 347-351.

Fine J. 1998. Le soutènement des galeries minières. Presses de l'École des Mines Paris.

Hagan PC. 2004. Variation in load transfer of a fully encapsulated rockbolt. In: Proceedings of the 23rd International Conference on Ground Control in Mining, Morgantown (USA), pp. 242-249.

Hyett AJ, Bawden WF, Macsporran GR, Moosavi M. 1995. A constitutive law for bond failure of fully-grouted cable bolts using a modified Hoek cell. Int J Rock Mech Min Sci 32: 11-36.

Ivanovic A, Neilson RD. 2009. Modelling of debonding along the fixed anchor length. Int J Rock Mech Min Sci 46: 699-707.

Kaiser P, Yazici S, Nosé J. 1992. Effect of stress change on the bond strength of fully grouted cables. Int J Rock Mech Min Sci 29: 293-306.

Laigle F. 2004. Modèle conceptuel pour le développement de lois de comportement adaptées à la conception des ouvrages souterrains. Mémoire de thèse, École Centrale de Lyon (France).

Launay J. 2010. Approche du fonctionnement et du dimensionnement d'une voûte de tunnel renforcée par boulons scellés et béton projeté. Tunnels et Espaces Souterrains 219: 184-193.

Li C, Stillborg B. 1999. Analytical models for rock bolts. Int J Rock Mech Min Sci 36: 1013-1029.

Moosavi M, Jafari A, Khosravi A. 2005. Bond of cement grouted reinforcing bars under constant radial pressure. Cem Concr Compos 27: $103-109$.

Offner C, Galvin J, Fabjanczyk M. 2000. Evaluating anchoring mechanisms of fully encapsulated rock bolts. In: Proceedings of the 19th International Conference on Ground Control in Mining, Morgantown (USA), pp. 255-260.

Pakalnis R, Peterson DA, Mah GP. 1994. Glass fibre cable bolts - an alternative. Bull Can Inst Min Metall 87: 53-57.

Popov EP. 1978. Mechanics of materials. Prentice Hall, NJ.

Reichert RD, Bawden WF, Hyett AJ. 1992. Evaluation of design bond strength for fully grouted bolts. Bull Can Inst Min Metall 85: 110-118.

Ren FF, Yang ZJ, Chen JF, ChenWW. 2010. An analytical analysis of the full-range behaviour of grouted rockbolts based on a tri-linear bond-slip model. Constr Build Mater 24: 361-370.

Reynolds C. 2006. Testing and standards for rock reinforcement consumables. Report 411, RMT Ltd. (for the UK Health and Safety Executive).

Saeb S, Amadei B. 1992. Modelling rock joints under shear and normal loading. Int J Rock Mech Min Sci 29: 267-278.

Stillborg B. 1986. Professional users handbook for rock bolting. Trans Tech Publications, Clausthal.

Tincelin E, Fine J. 1991. Mémento du boulonnage. Presses de l'École des Mines, Paris.

Verderame GM, Ricci P, De Carlo G, Manfredi G. 2009. Cyclic bond behaviour of plain bars. Part I: experimental investigation. Constr Build Mater 23: 3499-3511.

Windsor CR, Thompson AG. 1993. Rock reinforcement - Technology, testing, design and evaluation. Comprehensive rock engineering. Pergamon Press, Oxford, 451-484.

Yacizi S, Kaiser PK. 1992. Bond strength of grouted cable bolts. Int $J$ Rock MechMin Sci 29: 279-292.

Cite this article as: Laura Blanco-Martín, Michel Tijani, Faouzi Hadj-Hassen, Aurélien Noiret. Boulonnage à ancrage réparti : étude de l'interface barre-scellement sous sollicitations axiales. Rev. Fr. Geotech. 2016, 146, 2. 